\title{
The invasive red-eared slider turtle is more successful than the native Chinese three-keeled pond turtle: evidence from the gut microbiota
}

\author{
Yan-Fu Qu ${ }^{1}$, Yan-Qing Wu ${ }^{2}$, Yu-Tian Zhao ${ }^{1}$, Long-Hui Lin ${ }^{3}$, Yu Du ${ }^{1,4}{ }^{1,}$ Peng Li ${ }^{1}$, Hong Li ${ }^{1}$, Xiang Ji ${ }^{\text {Corresp. } 1}$ \\ 1 Jiangsu Key Laboratory for Biodiversity and Biotechnology, College of Life Sciences, Nanjing Normal University, Nanjing, Jiangsu, China \\ 2 Key Laboratory of Biosafety, Nanjing Institute of Environmental Sciences, Ministry of Ecology and Environment, Nanjing, Jiangsu, China \\ 3 Hangzhou Key Laboratory for Ecosystem Protection and Restoration, College of Life and Environmental Sciences, Hangzhou Normal University, \\ Hangzhou, Zhejiang, China \\ 4 Hainan Key Laboratory of Herpetological Research, College of Fisheries and Life Science, Hainan Tropical Ocean University, Sanya, Hainan, China \\ Corresponding Author: Xiang Ji \\ Email address: jixiang@njnu.edu.cn
}

Background: The mutualistic symbiosis between the gut microbial communities (microbiota) and their host animals has attracted much attention. Many factors potentially affect the gut microbiota, which also varies among host animals. The native Chinese three-keeled pond turtle (Chinemys reevesii) and the invasive red-eared slider turtle (Trachemys scripta elegans) are two common farm-raised species in China, with the latter generally considered a more successful species. However, supporting evidence from the gut microbiota has yet to be collected.

Methods: We collected feces samples from these two turtle species raised in a farm under identical conditions, and analyzed the composition and relative abundance of the gut microbes using bacterial $16 \mathrm{~S}$ rRNA sequencing on the Roach/454 platform.

Results: The gut microbiota was mainly composed of Bacteroidetes and Firmicutes at the phylum level, and Porphyromonadaceae, Bacteroidaceae and Lachnospiraceae at the family level in both species. The relative abundance of the microbes and gene functions in the gut microbiota differed between the two species, whereas alpha or beta diversity did not. Microbes of the families Bacteroidaceae, Clostridiaceae and Lachnospiraceae were comparatively more abundant in C. reevesii, whereas those of the families Porphyromonadaceae and Fusobacteriaceae were comparatively more abundant in $T$. s. elegans. In both species the gut microbiota had functional roles in enhancing metabolism, genetic information processing and environmental information processing according to the Kyoto Encyclopedia of Genes and Genomes database. The potential to gain mass is greater in $T$. s. elegans than in C. reevesii, as revealed by the fact that the Firmicutes/Bacteroidetes ratio was lower in the former species. The percentage of human disease-related functional genes was lower in $T$. s. elegans than in C. reevesii, presumably suggesting an enhanced potential to colonize new habitats in the former species. 


\section{The invasive red-eared slider turtle is more successful than the}

2 native Chinese three-keeled pond turtle: evidence from the gut

3 microbiota

5 Yan-Fu Qu ${ }^{1}$, Yan-Qing $\mathrm{Wu}^{2}$, Yu-Tian $\mathrm{Zhao}^{1}$, Long-Hui $\mathrm{Lin}^{3}, \mathrm{Yu} \mathrm{Du}^{1,4}$, Peng $\mathrm{Li}^{1}$,

6 Hong $\mathrm{Li}^{1}$ and Xiang $\mathrm{Ji}^{1^{*}}$

$8{ }^{1}$ Jiangsu Key Laboratory for Biodiversity and Biotechnology, College of Life Sciences,

9 Nanjing Normal University, Nanjing, Jiangsu, China

$10{ }^{2}$ Key Laboratory of Biosafety, Nanjing Institute of Environmental Sciences, Ministry of

11 Ecology and Environment, Nanjing, Jiangsu, China.

$12{ }^{3}$ Hangzhou Key Laboratory for Ecosystem Protection and Restoration, College of Life

13 and Environmental Sciences, Hangzhou Normal University, Hangzhou, Zhejiang, China

$14{ }^{4}$ Hainan Key Laboratory of Herpetological Research, College of Fisheries and Life

15 Science, Hainan Tropical Ocean University, Sanya, Hainan, China

17 Running head: Fecal microbiota in turtles

19 Corresponding author

20 Xiang Ji, jixiang@njnu.edu.cn

The manuscript for consideration as a Research Paper in PeerJ

October 2, 2020 


\section{ABSTRACT}

Background: The mutualistic symbiosis between the gut microbial communities (microbiota) and their host animals has attracted much attention. Many factors potentially affect the gut microbiota, which also varies among host animals. The native Chinese three-keeled pond turtle (Chinemys reevesii) and the invasive red-eared slider turtle (Trachemys scripta elegans) are two common farm-raised species in China, with the latter generally considered a more successful species. However, supporting evidence from the gut microbiota has yet to be collected.

Methods: We collected feces samples from these two turtle species raised in a farm under identical conditions, and analyzed the composition and relative abundance of the gut microbes using bacterial 16S rRNA sequencing on the Roach/454 platform.

Results: The gut microbiota was mainly composed of Bacteroidetes and Firmicutes at the phylum level, and Porphyromonadaceae, Bacteroidaceae and Lachnospiraceae at the family level in both species. The relative abundance of the microbes and gene functions in the gut microbiota differed between the two species, whereas alpha or beta diversity did not. Microbes of the families Bacteroidaceae, Clostridiaceae and Lachnospiraceae were comparatively more abundant in C. reevesii, whereas those of the families Porphyromonadaceae and Fusobacteriaceae were comparatively more abundant in T. s. elegans. In both species the gut microbiota had functional roles in 
44 enhancing metabolism, genetic information processing and environmental information 45 processing according to the Kyoto Encyclopedia of Genes and Genomes database. The 46 potential to gain mass is greater in T. s. elegans than in C. reevesii, as revealed by the fact

47 that the Firmicutes/Bacteroidetes ratio was lower in the former species. The percentage

48 of human disease-related functional genes was lower in T. s. elegans than in C. reevesii,

49 presumably suggesting an enhanced potential to colonize new habitats in the former 50 species.

51 Keywords Gut microbiota, Gut microbial diversity, Invasive species, Native species,

52 Chinemys reevesii, Trachemys scripta elegans, Turtle 


\section{INTRODUCTION}

55 The past few years have witnessed much attention paid to the gut microbiota of aquatic 56 animals including invertebrates (Meziti, Mente \& Kormas, 2012; Wang et al., 2019; Gao

57 et al., 2020), fish (Divya et al., 2012; Xing et al., 2013; Egerton et al., 2018), reptiles

58 (Ahasan et al., 2018; Zhang et al., 2019; Scheelings et al., 2020) and mammals (Nelson et al., 2013; Delport et al., 2016). The gut microbes have a mutually-beneficial relationship with their hosts. Numerous studies have confirmed that the gut microbes encode 10 to 100-fold more distinct genes than their host genome (Turnbaugh et al., 2006; Qin et al., 2010), and that they are involved in a large number of host biological processes related to nutrient absorption (Kartzinel et al., 2019), gut homeostasis maintenance (Buchon, Broderick \& Lemaitre, 2013), growth (Videvall et al., 2019) and even behavioral expression (Ye et al., 2014). Therefore, it is of great significance to study the coevolutionary relationship between the gut microbes and their hosts. and sea lions (Delport et al., 2016), and Proteobacteria and Firmicutes in sharks (Givens et al., 2015). Each gut microbial phylum may have unique functional roles. For example, 
74

members of the phylum Proteobacteria contribute to the breakdown and ferment of the complex sugars and are related to the biosynthesis of vitamins for their hosts (Colston \& Jackson, 2016), microbes of the phylum Bacteroidetes improve the digestive efficiency in both herbivorous and carnivorous species by degrading the complex macromolecular matter (Colston \& Jackson, 2016), members of the phylum Firmicutes contribute to the production of enzymes involved in fermenting vegetative material and have the potential to fabricate vitamin B (Rowland et al., 2018), and microbes of the phylum Tenericutes are involved in the nutrient processing of their hosts (Colston \& Jackson, 2016). Taken together, gut microbes play a vital role in maintaining the normal life of the host.

Gut microbes are affected by many factors, including the host's genetic background, food habit (Kohl et al., 2017), ontogenetic stage (Videvall et al., 2019), gender (Mueller et al., 2006) and health status (Lin et al., 2019), and vary seasonally (Kohl et al. 2017; Kartzinel et al., 2019). The phylogenetic dependence of the gut microbial communities has been documented in fish (Givens et al., 2015), reptiles (Hong et al., 2011), birds (Capunitan et al., 2020) and mammals (Kartzinel et al., 2019). In mammals, for example, the gut microbial diversity is higher in herbivorous species than in carnivorous species (Kartzinel et al., 2019). In humans, microbes of the phylum Firmicutes are positively correlated with latitude and obesity, whereas members of the phylum Bacteroidetes show opposite correlations (Ley et al., 2005; Suzuki \& Worobey, 2014). Moreover, the 
94 gut microbial profiles at the different life stages are complicated by the natural

95

96 dynamics and complex interactions between intrinsic and extrinsic factors. For example, early growth, household location, and antibiotic experiences during pregnancy are correlated with the early gut microbial composition in humans (Vatanen et al., 2019). Seasonal variation in diets may lead to changes in the gut microbiota (Kartzinel et al., 2019), and exposure of host animals to artificial environments (e.g., animals in captivity) may lead to an increase in the abundance of human disease-related functional genes in the gut microbes (Tang et al., 2020; Zhou et al., 2020). Taken together, the gut microbial community is affected by the host's genetic background, diets and individual status and, in turn, affects the physiological, behavioral and even evolutionary processes of the host. In the present study, we compared gut microbes between the native Chinese threekeeled pond turtle (Chinemys reevesii) and the invasive red-eared slider turtle (Trachemys scripta elegans) using bacterial 16S rRNA sequencing on the Roach/454 platform. Both are well studied species and among common turtle species farm-raised in China as food, pets and traditional medicine, with T. s. elegans well known as a very successful invasive species in many places around the world (Cadi et al., 2004). The red-eared slider turtle is believed to be introduced to mainland China through Hong Kong as a pet and source of food in the 1980s and has become established in many parts of China (Ma \& Shi, 2017). The turtle is generally considered to be more successful than native freshwater turtles such as the Chinese three-keeled pond turtle and has be listed by the 
114 World Environmental Protection Organization as one of the 100 most harmful invasive

115 species (Ma \& Shi, 2017). However, supporting evidence from the gut microbiota has

116 yet to be collected.

117

118 MATERIALS AND METHODS

119 Sample collection

120 This study was performed in accordance with the current laws on animal welfare and

121 research in China, and was approved by the Animal Research Ethics Committee of

122 Nanjing Normal University (IACUC-200422).

123 We obtained three juvenile C. reevesii and three juvenile T. s. elegans in March 2013

124 from a turtle farm in Hangzhou, Zhejiang, East China, and brought them to our

125 laboratory in Nanjing, where they were and individually housed in $340 \times 230 \times 200 \mathrm{~mm}$

126 (length $\times$ width $\times$ height) aquariums placed in a room for six months. Water

127 temperatures varied from $26-30^{\circ} \mathrm{C}$ (with a mean of $28^{\circ} \mathrm{C}$ ), and photoperiod inside the

128 room was on a cycle of $12 \mathrm{~h}$ light and $12 \mathrm{~h}$ dark. Turtles were fed with commercially

129 sold food (10\% water, 60\% proteins, 5\% lipids, 5\% carbohydrates and 20\% minerals; Li

130 et al., 2013) at an average ration of $1.5 \%$ body mass daily. At the end of the experiment,

131 body masses varied from 56-70 g (with a mean of $64 \mathrm{~g}$ ) in C. reevesii, and from 118-136

$132 \mathrm{~g}$ (with a mean of $125 \mathrm{~g}$ ) in T. s. elegans. We collected fecal samples from each turtle on

$13325^{\text {th }}$ September 2013 and stored them at $-80^{\circ} \mathrm{C}$ until later DNA extraction. 


\section{Sample sequencing}

135 Genomic DNA was extracted using the Qiagen TM QIAamp DNA Stool Mini Kit

136 (Hilden, Germany) following the manufacturer's instructions. The V1-V3 region of the

137

138

139

140

141

142

143

144

145

146

147

148

149

150

151

152

153

16S rRNA gene was chosen for the amplification and subsequent pyrosequencing of the PCR products. The following $16 \mathrm{~S}$ rRNA primers were used for the PCR reaction: $8 \mathrm{~F}$ (5'AGAGTTTGATCCTGGCTCAG - 3') and 533R (5' - TTACCGCGGCTGCTGGCAC- 3’) for the V1-V3 regions. Each primer included "barcode" sequences to facilitate the sequencing of products in the Roche/454 GS FLX+ system (454 Life Sciences, USA). The fusion primer sequences were 5'-454adapter-mid- CCTACGGGAGGCAGCAG-3' (forward) and 5'-454adapter-mid- CCGTCAATTCMTTTRAGT-3' (reverse). DNA (20 ng) from each sample was used for amplification in $25 \mu$ reactions that contained $2.5 \mu 1$ 10fold reaction buffer, $40 \mathrm{ng}$ of fecal DNA, $10 \mu \mathrm{M}$ each primer, $0.625 \mathrm{U}$ Pyrobest polymerase (Takara), and $5000 \mu \mathrm{M}$ concentration of each of four deoxynucleoside triphosphates. PCR reactions were started by an initial denaturation at $94{ }^{\circ} \mathrm{C}$ for $5 \mathrm{~min}$ followed by 27 amplification cycles $\left(94^{\circ} \mathrm{C}\right.$ for $30 \mathrm{~s}, 45 \mathrm{~s}$ at annealing temperature, $72{ }^{\circ} \mathrm{C}$ for $1 \mathrm{~min}$ ) and a final extension step for $7 \mathrm{~min}$ at $72{ }^{\circ} \mathrm{C}$. Subsequently, PCR products were examined for size and yield using agarose gel in TAE buffer (20 mM Tris- $\mathrm{HCl}, 10$ mM sodium acetate, $0.5 \mathrm{mM} \mathrm{Na}{ }_{2}$ EDTA, $\mathrm{pH} 8.0$ ). Quantification of the PCR products was performed by using the PicoGreen dsDNA BR assay kit as recommended by the manufacturer. Then, the V1-V3 region of $16 \mathrm{~S}$ rRNA was sequenced on a Roche GS-FLX 
154454 platform (Roche, Shanghai, China) according to 454 protocols.

\section{Quality control and data standardization}

156 The pyrosequencing data were optimized by control standards according to the

157

158

159

160

161

162

163

164

165

166

167

following criteria: (1) the raw reads must perfectly match the primers we used; (2) the barcode sequences with quality average were at least $25 \%$; (3) the range of read length was between 320 to 800 bp nucleotides except for barcodes and primers; (5) consecutively identical bases did not exceed six and excluding undetermined bases. A total of 52,469 high-quality reads with an average length of 480 bp nucleotides were obtained. These sequences were then submitted to the National Center for Biotechnology Information (NCBI) Bioproject database (SRA accession number PRJNA645767).

We used Usearch 7.0 to conduct analysis of the operational taxonomic units (OTUs) (Edgar, 2010). We first extracted nonrepeative sequences from high-quality data to reduce redundant calculations in the analysis and removed the singletons. Then, these available sequences were clustered into OTUs according to the similarity criteria of $97 \%$, and chimeras were removed in the clustering process to obtain the representative sequences of OTUs. Finally, map all available sequences for each sample to representative sequences of OTUs to obtain OTU tables for further analysis. Moreover, RDP Classifier 2.2 (Quast et al., 2012) performed a taxonomic analysis of the OTU representative sequences against the Greengenes 135/16S Database at the confidence 
174 threshold of $70 \%$ to obtain the taxonomic information corresponding to each OTU. To

175 avoid large partial sample deviations, we retained OTUs with the number of OTU

176 greater than 5 in at least 2 samples for further analysis

177

The alpha diversity index under different random sampling was calculated by

178

179

180

181

182

183

184

185

186

187 using mothur 1.30.2 (Schloss et al., 2009) and visualized using the R 4.0.1 (R

Development Core Team, 2020) to assess the adequacy of sequencing data. We standardize the OTUs abundance information according to the sample with the least sequence number for further analysis.

\section{Alpha and beta diversity estimation}

The alpha-diversity indexes including the community richness (ACE index), diversity (Shannon diversity), evenness (Shannoneven index), and coverage (the Good's coverage index) were calculated using mothur software for each sample. These estimators were presented visually in the form of pictures using the R software platform. Bartlett test was used to test whether the data is of equal variance and the equal-variance t-test or the heteroscedasticity t-test was conducted to compare between species according to the Bartlett test results.

The principal component analysis (PCoA) and non-metric multidimensional scaling (NMDS) were conducted to test the differences in the relative abundance of OTUs of gut microbiome between the two species. The analysis of similarity test between the two species was conducted using ANOSIM based on the bray_curtis 
194 distance with 999 permutations. The PCoA and NMDS analyses were performed using

195 the package vegan in R (Oksanen et al., 2013). Moreover, the linear discriminant

196 analysis effect size (LEfSe) (Segata et al., 2011) was conducted to test the differences in

197 the bacterial abundances from phylum to family between the two species. Also, the

198 linear discriminatory analysis (LDA) was conducted to estimate the effect size for each

199 selected classification. In this study, only the bacterial taxa with a log LDA score $>4$

200 (over 4 orders of magnitude) were used. LEfSe and LDA analyses were performed

201 using the Galaxy online tools (Afgan et al., 2018).

202

203

204

205

206

207

208

209

210

211

212

213

\section{Gene function prediction}

We used PICRUSt to search the GreenGene ID of the corresponding OTU based on the Kyoto Encyclopedia of Genes and Genomes (KEGG) database (Kanehisa, 2019) and to predict gene functions. Further, these function genes were classified and assigned to the relevant KEGG pathways (Langille et al., 2013). We calculated the numbers of functional genes in each pathway to compare the functional enrichment in gut microbiota between the two species. The Circos diagram was plotted to show the relative abundance and distribution status of KOs genes between the two species. We performed the LEfSe to compare the abundances of function genes from KOs gene-level 1 to level 3 between the two species, thereby determining their differences in gene functions. Moreover, LDA analysis was used to assess the effect size for each three KO gene levels. In this study, only gene functions with a log LDA score $>2$ were used. 
214 Student's t-test was used to compare the relative abundance of the KOs gene at different

215 levels. All values were presented as mean \pm SE, and all statistical analyses were

216 conducted at the significance level of $\alpha=0.05$.

217

218 RESULTS

219 Gut microbial profile

220 We obtained 31,402 raw reads from C. reevesii and 31,193 raw reads from T. s. elegans.

221 After quality control and filtration, we harvested 27,945 and 24,519 high-quality

222 sequences from $C$. reevesii and T. s. elegans, respectively. The average read was 8,745 \pm 399

223 (ranging from 7605-10751 reads per sample) with a sequence length of $480 \pm 4 \mathrm{bp}$

224 (ranging from 326-635 bp) for each sample (Fig. S1). Further, an average of 9,317 \pm 601

225 high-quality reads (ranging from 8,308-10,751 reads per sample) and 8,173 \pm 242 high-

226 quality reads (ranging from 7,605-8,603 reads per sample) were obtained from $C$. reevesii

227 and T. s. elegans, respectively. The rarefaction curves based on the Shannon index for all

228 samples showed that sufficient sequence numbers could be obtained for further analysis

229 at the current sequencing depth (Fig. S2). Furthermore, the minimum values of the

230 Good's coverage were more than $99.8 \%$, which meant that the vast majority of gut

231 bacteria could be retrieved from these samples.

232 We obtained 183 representative sequences of OTUs at the 97\% similarity level, and

23380 representative sequences after removing the influence of OTUs with a large 
234 deviation. Among them, the average OTUs were $29 \pm 1.7$ (ranging from 25-32) and $33 \pm 2.9$

235 (ranging from 29-40) per sample for C. reevesii and T. s. elegans, respectively (Table S1).

236 Fifty-four OTUs were shared by the two species, three were unique to T. s. elegans, and

23723 were unique to C. reevesii. These OTUs were clustered into five phyla, seven classes,

238 seven orders, 17 families and 28 genera based on phylogenetic classification for the six

239 fecal samples (Table S1). More specifically, species in the gut microbiota belonged to

240 four families, five classes, six orders, 10 families and 10 genera in C. reevesii, and to five

241 phyla, seven classes, seven orders, 17 families and 28 genera in T. s. elegans (Table S1).

242 Interspecific differences in the gut microbiota

243 As the unidentified OTUs accounted for up to $15.1 \%$ at the family level and $41.6 \%$ at the

244 genus, we analyzed the composition and relative abundance of gut bacterial community

245 excluding data at the genus level. Figure 1 shows the relative abundances of the gut

246 microbiota across taxonomic levels from family to phylum. Considering all six samples

247 as a whole, we found that the fecal microbiota was dominated by species of the phyla

248 Bacteroidetes $(78.02 \pm 8.00 \%)$, Firmicutes $(20.21 \pm 7.94 \%)$, Proteobacteria $(1.46 \pm 0.98 \%)$ and

249 Fusobacteria $(0.31 \pm 0.14 \%)$, the classes Bacteroidia $(78.02 \pm 8.00 \%)$, Clostridia

250 (19.73 $\pm 7.85 \%)$, Gammaproteobacteria (1.46 $\pm 0.98 \%)$, Erysipelotrichi $(0.48 \pm 0.13 \%)$ and

251 Fusobacteriia $(0.31 \pm 0.14 \%)$, the orders Bacteroidales $(78.02 \pm 8.00 \%)$, Clostridiales

252 (19.73 $\pm 7.85 \%)$, Erysipelotrichales (1.87 $\pm 1.01 \%)$, Fusobacteriales $(0.31 \pm 0.14 \%)$ and

253 Aeromonadales $(0.07 \pm 0.03 \%)$, and the families Porphyromonadaceae $(43.88 \pm 8.83 \%)$, 


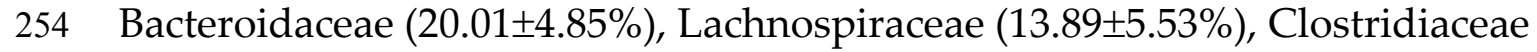

255 (2.41 $\pm 0.91 \%)$, S24-7 (1.77 $\pm 1.15 \%)$, Enterobacteriaceae (1.39 $\pm 0.99 \%)$, Ruminococcaceae

$256(0.66 \pm 0.09 \%)$, Erysipelotrichaceae $(0.46 \pm 0.13 \%)$, Fusobacteriaceae $(0.31 \pm 0.14 \%)$ and

257 Aeromonadaceae $(0.07 \pm 0.03 \%)$.

258 The composition and relative abundance of the gut microbiota at different

259 taxonomic levels showed differences between the two species. For the sake of

260 convenience, the bacteria with a relative abundance $>2 \%$ were defined as the dominant

261 taxa. The dominant phyla were Bacteroidetes (63.45 $\pm 10.38 \%)$, Firmicutes $(34.12 \pm 10.75 \%)$

262 and Proteobacteria $(2.40 \pm 1.80 \%)$ in C. reevesii, and Bacteroidetes $(92.59 \pm 2.63 \%)$ and

263 Firmicutes $(6.30 \pm 2.77 \%)$ in T. s. elegans (Fig. 1A). The dominant classes were Bacteroidia

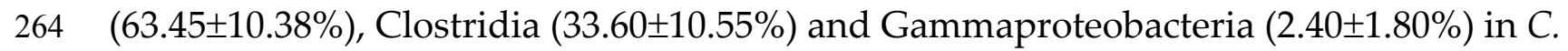

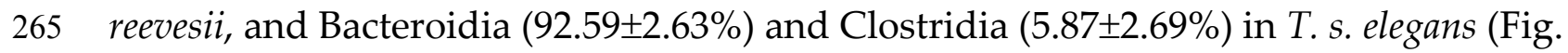

266 1B). The dominant orders were Bacteroidales $(63.45 \pm 10.38 \%)$, Clostridiales

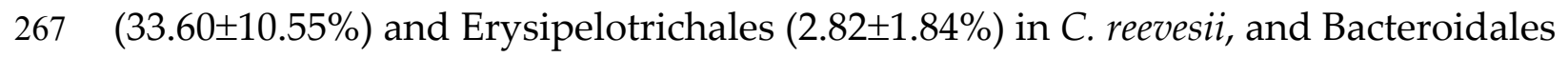

268 (92.59 $\pm 2.63 \%)$ and Clostridiales (5.87 $\pm 2.69 \%)$ in T. s. elegans (Fig. 1C). The dominant

269 families were Bacteroidaceae (30.74 $\pm 2.95 \%)$, Porphyromonadaceae $(26.77 \pm 10.33 \%)$,

270 Lachnospiraceae (3.81 $\pm 1.92 \%)$, Clostridiaceae (3.87 $\pm 1.27 \%)$, S24-7 $(3.22 \pm 1.98 \%)$ and

271 Enterobacteriaceae (2.29 $\pm 1.84 \%)$ in C. reevesii, and Porphyromonadaceae $(60.99 \pm 3.11 \%)$,

272 Bacteroidaceae (9.28 $\pm 2.97 \%)$ and Lachnospiraceae (3.81 $\pm 1.92 \%)$ in T. s. elegans (Fig. 1D).

273 Microbes of the phylum Firmicutes, the class Clostridia, the order Clostridiales and 
274 the families Bacteroidaceae, Clostridiaceae and Lachnospiraceae were more abundant in

275 C. reevesii (Table S3; Fig. 2). Microbes of the phyla Bacteroidetes and Fusobacteria, the

276 classes Bacteroidia and Fusobacteriia, the orders Fusobacteriales and Bacteroidales and

277 the families Porphyromonadaceae and Fusobacteriaceae were more abundant in T.s.

278 elegans (Table S3; Fig. 2).

279 Table S2 shows the alpha-diversity indexes for each sample including the

280 community richness (ACE index), diversity (Shannon diversity), evenness

281 (Shannoneven index), and coverage (the Good's coverage index). Student's $t$ test

282 showed that none of these indexes differed between the two species (all $P>0.09$ ). PCoA

283 and NMSD analyses showed no significant differences in bacterial relative abundance

284 between the two species (Fig. 3; anosim: $R=0.44, P=0.10$ ).

\section{The predicted metagenomes}

286 PICRUSt analysis was performed to predict the gene functions in the two turtle species

287 based on the 16S RNA of six fecal samples. These gene functions were predicted into

288 three levels of KEGG functional categories. Among them, metabolism-related genes had

289 an overwhelming proportional advantage, with a relative abundance of up to

$29045.34 \pm 1.38 \%$ at the first level (Fig. 4A). Furthermore, the gene functions at the first level

291 also included environmental information processing (17.12 $\pm 1.43 \%)$, genetic information

292 processing $(16.21 \pm 0.45 \%)$, human diseases $(15.80 \pm 0.43 \%)$, cellular processes $(4.03 \pm 0.33 \%)$,

293 organismal systems $(0.59 \pm 0.06 \%)$ and unclassified genes $(15.80 \pm 0.43 \%)$ (Fig. 4A). There 
294 were 18 major gene functions at the second level ( Fig. 4B), among which, the most

295 abundant gene functions were composed of membrane transport $(14.64 \pm 1.30 \%)$,

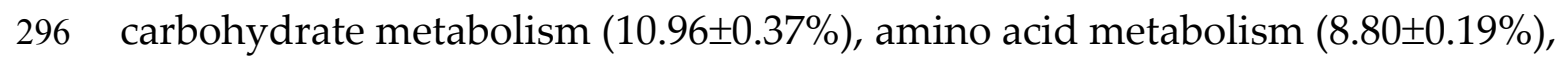

297 replication and repair (6.94 $\pm 0.23 \%)$, energy metabolism (5.00 $\pm 0.20 \%)$, translation

$298(4.02 \pm 0.18 \%)$, metabolism of cofactors and vitamins $(3.79 \pm 0.05 \%)$, cell motility

299 (3.37 $\pm 0.42 \%)$, nucleotide metabolism (3.26 $\pm 0.13 \%)$ and transcription $(3.06 \pm 0.08 \%)$ (Fig.

$3004 \mathrm{~B})$. Also, at the third level, the gene functions with the highest relative abundance were

301 transporters $(7.18 \pm 0.56 \%)$, ABC transporters $(3.77 \pm 0.40 \%)$, DNA repair and

302 recombination proteins $(2.25 \pm 0.05 \%)$, transcription factors $(2.21 \pm 0.16 \%)$ and two-

303 component system (2.19 $\pm 0.19 \%)$ (Figs. 4C and S3).

304 As a whole, 240 known KOs and 27 unknown gene functions were identified from

305 the six samples. The distribution of KOs in different species with their relative

306 abundance of $>1 \%$ was displayed on the Circos diagram (Fig. S3). The gene functions at

307 the first level that accounted for the highest abundance included metabolism

$308(46.76 \pm 2.45 \%)$, genetic information processing $(16.84 \pm 0.60 \%)$ and environmental

309 information processing (15.48 $\pm 2.51 \%)$ in C. reevesii, and metabolism $(43.91 \pm 0.54 \%)$,

310 environmental information processing $(18.76 \pm 0.30 \%)$ and genetic information

311 processing $(15.59 \pm 0.44 \%)$ in T. s. elegans (Figs. 4A and S3). The relative abundance of

312 gene functions at the second level accounted more than 5\% were membrane transport

$313(13.13 \pm 2.27 \%)$, replication and repair $(7.32 \pm 0.29 \%)$, amino acid metabolism $(8.93 \pm 0.31 \%)$, 
314 carbohydrate metabolism $(11.41 \pm 0.58 \%)$, and energy metabolism $(5.26 \pm 0.33 \%)$ in C.

315 reevesii, and those in T. s. elegans were membrane transport $(16.15 \pm 0.16 \%)$, replication

316 and repair $(6.56 \pm 0.18 \%)$, amino acid metabolism $(8.67 \pm 0.17 \%)$ and carbohydrate

317 metabolism $(10.51 \pm 0.27 \%)$ (Figs. $4 \mathrm{~B}$ and S3). Furthermore, transporters $(6.66 \pm 0.13 \%$ in $C$.

318 reevesii, and $7.69 \pm 0.15 \%$ in $T$. s. elegans) and ABC transporters $(3.28 \pm 0.68 \%$ in C. reevesii,

319 and $4.27 \pm 0.15 \%$ in T. s. elegans) at the third level accounted more than $3 \%$ in both species

320 (Figs. 4C and S3).

321 LEfSe analysis based on the KOs revealed obvious differences in gene functions

322 between the two species. At the third level, LDA discriminant analysis showed that

323 there was a greater proportion of human disease-related functions (e.g. valine, leucine,

324 and isoleucine biosynthesis, and Influenza A, both $P=0.046$ ) and metabolism-related

325 functions (e.g. bisphenol degradation, linoleic acid metabolism, nitrogen metabolism

326 and colorectal cancer, all $P=0.050)$ in $C$. reevesii, as well as tyrosine metabolism $(L D A=$

327 2.79562, $P=0.050)$ in T. s. elegans.

\section{DISCUSSION}

329 From this study where the gut microbiota was compared between two species of turtles

330 raised under the identical conditions for six months we knew the following. First,

331 neither alpha diversity nor beta diversity differed significantly between the two species

332 (Table S2; Fig. 3). Second, the relative abundance of gut microbes differed significantly 
333 between the two species across the taxonomic levels from family to phylum (Fig. 2).

334 Third, the relative abundance of metabolism-related functional genes in the gut 335 microbiota differed significantly between the two species, and so did the relative 336 abundance of human disease-related functional genes (Fig. 4).

337 The influence of the host's genetic background on the gut microbiota has been 338 detected in most vertebrates studied thus far, including fish, reptiles, birds and 339 mammals. For instance, phylogenetic relationships shape the gut microbial profile in 340 phylogenetically closely related mammals that are similar in body shape, craniofacial anatomy and the gut structure (Ley et al., 2008; Kartzinel et al., 2019. The taxonomic or phylogenetic correlates of the gut microbiota has also been reported for birds in Equatorial Guinea (Capunitan et al., 2020). In Galápagos iguanas, differences in the fecal microbial communities are primarily related to the host species and ecotype, and subsequently to population origin (Lankau et al., 2012). Significant differences in the gut microbiota also exist between the bony fish and sharks (Givens et al., 2015). From these results we know that the influences of phylogeny on the gut microbiota are universal in vertebrates. This is probably the main reason for why we could still distinguish differences in the gut microbiota between two species of turtles even though they had been maintained under identical conditions for six months. Bacteroidetes and Firmicutes were two dominant phyla in the gut microbiota in 
353 the gopher tortoise Gopherus polyphemus (Yuan et al., 2015), the green sea turtle

354 Chelonia mydas (Campos et al., 2018; McDermid et al., 2020), and the loggerhead sea

355 turtle Caretta caretta (Arizza et al., 2019), and the painted turtle Chrysemys picta (Fugate

356 et al., 2020). Gut microbes of the families Bacteroidaceae, Clostridiaceae and

357 Lachnospiraceae were comparatively more abundant in C. reevesii than in T. s. elegans

358 (Fig. 1). Of these three families, Bacteroidaceae belongs to the phylum Bacteroidetes,

359 and Clostridiaceae and Lachnospiraceae both belong to the order Clostridiales, the class

360 Clostridia and the phylum Firmicutes. Furthermore, gut microbes of the families

361 Porphyromonadaceae and Fusobacteriaceae were more abundant in T. s. elegans than in

362 C. reevesii (Fig. 2). Porphyromonadaceae belongs to the order Bacteroidales, the class

363 Bacteroidia and the phylum Bacteroidetes, and Fusobacteriaceae belongs to the order

364 Fusobacteriales, the class Fusobacteriia and the phylum Fusobacteria (Fig. 2). Microbes

365 of the families Bacteroidaceae and Porphyromonadaceae were the main components of

366 the phylum Bacteroidetes (Fig. 2). Bacteroidetes species in the gut play an important

367 role in degrading carbohydrates and proteins, thus being essential for their hosts in the

368 absorption and utilization of nutrients (Fernando et al., 2010; Nuriel-Ohayon, Neuman

369 \& Koren, 2016). Firmicutes species are universal representatives in mammalian gut

370 microbiota and play important functional roles in digestion and host metabolism (Ley

371 et al., 2008). Fusobacteria is usually rare in the gut microbiota of reptiles, but can be

372 obtained from the infected animals and are the dominant phylum in the gut microbiota 
373 in scavengers like the American alligator Alligator mississippiensis (Keenan, Engel \&

374 Elsey, 2013), the black vulture Coragyps atratus and the turkey vulture Cathartes aura

375 (Mendoza et al., 2018). The Firmicutes/Bacteroidetes ratio was 0.54 in C. reevesii and 0.07

376 in T. s. elegans. It is worth noting that the Firmicutes/Bacteroidetes ratio is negatively

377 correlated with mass gain (Fernando et al., 2010). Thus, a lower

378 Firmicutes/Bacteroidetes ratio in T. s. elegans suggests a greater potential to gain mass in

379 the species.

380 Numerous studies on aquatic animals have shown that the gut microbiota is

381 influenced by food ingested, environment, and their age, gender and health status. For

382 example, colony location and captivity influence the gut microbial community composition of the Australian sea lion Neophoca cinerea (Delport et al., 2016). The gut microbial diversity in green turtles are influenced by their age and population origin

(Campos et al., 2018). Likewise, the gut microbiota of fish is affected by life stage, tropic

level, diet, season, habitat, sex, captivity and phylogeny (Egerton et al., 2018). Our experiment design allowed us to exclude the effects of external factors on the gut microbiota because turtles we used were raised under the same conditions for a half year.

Neither alpha diversity nor beta diversity differed between the two species (Table S2, Fig. 3). This might be related to small sample sizes in both species. Data from one $C$. reevesii sample deviated from those from other samples, suggesting that future work 
393 could usefully examine the impact of long-term homogeneous culture conditions on the 394 gut microbes in animals. The composition of gut microbes in the two species was 395 relatively simple, presumably because turtles were raised under identical conditions for a long time period (six months). Similar results have been reported in an earlier study of T. s. elegans where gut microbes in fecal samples from three adult turtles are mainly composed of Firmicutes and Bacteroidetes (Du, Zhang \& Shi, 2013). In that study, Bacteroides account for $98.82 \%$ of the Bacteroidetes, and Clostridium accounts for $95.81 \%$

of the Firmicutes (Du, Zhang \& Shi, 2013). In another study of T. s. elegans the gut microbiota in young turtles is mainly composed of Firmicutes, Bacteroidetes and

Proteobacteria (Peng et al., 2020).

Our data showed that in both species the most functionally distinct categories were focused on metabolism, genetic information processing and environmental information processing at the first function level, followed by gene functions associated with membrane transport, replication and repair, amino acid metabolism and carbohydrate metabolism at the second level, and transporters and $\mathrm{ABC}$ transporters at the third level. In reptiles, that the gene functions of the gut microbes are associated with metabolism has been reported for the timber rattlesnake Crotalus horridus (McLaughlin et al., 2015), the crocodile lizard Shinisaurus crocodilurus (Tang et al., 2020), and the northern grass

411 lizard Takydromus septentrionalis (Zhou et al., 2020). Similar results have also been 412 reported for birds (Wang et al., 2018) and mammals (Zhao et al., 2018). Previous studies 
413 generally support the idea that the most functionally distinct categories of gut microbes

414 play an important role in host energy metabolism.

415 Comparing gene functions between C. reevesii and T. s. elegans, we found that they

416 differed in human diseases and metabolic-related functions. Captive animals had an

417 ample opportunity to come into contact with human keepers for transmission of

418 microbiota from host-associated sources, which could colonize the animals. In this

419 study, we found that gut microbes of the families Bacteroidaceae, Clostridiaceae, and

420 Lachnospiraceae were more abundant in C. reevesii. Clostridiaceae and Lachnospiraceae

421 (Firmicutes; Clostridia; Clostridiales) are more abundant in the gut microbiota for the

422 time periods of disease progression (Zhang et al., 2014). The relative abundance of

423 Bacteroidaceae (Bacteroidetes; Bacteroidia; Bacteroidales) increases at one or two

424 months old in humans and is negatively correlated with depression (Songjinda et al.,

425 2007; Strandwitz et al., 2019). Gut microbes of the families Porphyromonadaceae and

426 Fusobacteriaceae were comparatively more abundant in T. s. elegans.

427 Porphyromonadaceae (Bacteroidetes; Bacteroidia; Bacteroidales) is negatively

428 associated with cognitive decline, affective disorders and anxiety-like behavior in aged

429 mice (Scott et al. 2017). Fusobacteriaceae (Fusobacteria; Fusobacteriia; Fusobacteriales)

430 is involved in the fermentative progress of a variety of carbohydrates, amino acids and

431 peptides (Zoqratt et al., 2018). Therefore, we hypothesized that the difference in gene

432 function between $C$. reevesii and T. scripta elegans may be related to their absorption and 
433 utilization of food resources. Comparative studies have found that C. reevesii is at

434 greater risk of colonizing human gut microbes than T. s. elegans, so it is recommended

435 that actions be taken to minimize direct contact between human managers and native

436 turtles.

437

438 CONCLUSION

439 Our data showed that red-eared slider turtles and Chinese three-keeled pond turtles

440 raised under identical conditions for a long time period (six months) differed in the

441 relative abundance of the microbes and gene functions in the gut microbiota, thus

442 adding evidence for the phylogenetic (genetic) dependence of the gut microbial

443 communities in reptiles. The potential to gain mass was greater in T. s. elegans than in $C$.

444 reevesii, as revealed by the fact that the Firmicutes/Bacteroidetes ratio was lower in the

445 former species. The percentage of human disease-related functional genes was lower in

446 T. s. elegans than in C. reevesii, presumably suggesting an enhanced potential to colonize

447 new habitats in the former species. Taken together, our data allow the conclusion that

448 the invasive red-eared slider turtle is more successful than the native Chinese three-

449 keeled pond turtle.

\section{ACKNOWLEDGEMENTS}

451 We would like to thank Zhe Liu and Rong-Fang Wang for help with feeding turtles. 
452 ADDITIONAL INFORMATION AND DECLARATIONS

\section{Funding}

454 The work was supported by grants from the Natural Science Foundation of Jiangsu

455 Province (BK20161556), the Priority Academic Program Development of Jiangsu Higher

456 Education Institutions (PAPD) of Jiangsu Higher Education Institutions and the Finance

457 Science and Technology Project of Hainan Province (ZDKJ2016009-1-2). The funders

458 had no role in the study design, data collection and analysis, decision to publish, or

459 preparation of the manuscript.

\section{Grant Disclosures}

461 The following grant information was disclosed by the authors:

462 The Natural Science Foundation of Jiangsu Province: BK20161556.

463 The Priority Academic Program Development (PAPD) of Jiangsu Higher Education

464 Institutions.

465 The Finance Science and Technology Project of Hainan Province: ZDKJ2016009-1-2.

\section{Competing Interests}

467 The authors declare that they have no competing interests.

\section{Author Contributions}

- Yan-Fu Qu conceived, designed and performed the experiments, analyzed the data, 
470 contributed reagents/materials/analysis tools, prepared figures and tables, wrote

471 the paper, reviewed drafts of the paper, and approved the final draft.

472

473

474

475

476

477

478

479

480

481

482

483

484

485

486

487

488

489

- Yan-Qing Wu performed the experiments, contributed reagent/materials/tools, wrote the paper, reviewed drafts of the paper, and approved the final draft.

- Yu-Tian Zhao performed the experiments, analyzed the data, prepared figures and tables, wrote the paper, reviewed drafts of the paper, and approved the final draft.

- Long-Hui Lin conceived and designed the experiments, wrote the paper, reviewed drafts of the paper, and approved the final draft.

- $\mathrm{Yu}$ Du performed the experiments, contributed reagent/materials/tools, reviewed drafts of the paper, and approved the final draft.

- Peng Li performed the experiments, contributed reagent/materials/tools, reviewed drafts of the paper, and approved the final draft.

- Hong Li performed the experiments, contributed reagent/materials/tools, reviewed drafts of the paper, and approved the final draft.

- Xiang Ji conceived, designed and supervised the experiments, contributed reagent/materials/tools, wrote the paper, reviewed drafts of the paper, and approved the final draft.

\section{Data Availability}

The following information was supplied regarding data availability:

Data are available at the National Center for Biotechnology Information (NCBI) 
490 Bioproject database (SRA accession number PRJNA645767).

491 Supplemental Information

492 Supplemental information for this article can be found online.

493

494

495

496

497

498

499

500

501

502

503

504

505

506

507

508

\section{REFERENCES}

Afgan E, Baker D, Batut B, Beek Mvd, Bouvier D, Čech M, Chilton J, Clements D, Coraor N, Grüning B, Guerler A, Hillman-Jackson J, Jalili V, Rasche H, Soranzo N, Goecks J, Taylor J, Nekrutenko A, Blankenberg D. 2018. The Galaxy platform for accessible, reproducible and collaborative biomedical analyses: 2018 update. Nucleic Acids Research 46:W537-W544 DOI10.1093/nar/gky379.

Ahasan MS, Waltzek TB, Huerlimann R, Ariel E. 2018. Comparative analysis of gut bacterial communities of green turtles (Chelonia mydas) pre-hospitalization and post-rehabilitation by high-throughput sequencing of bacterial $16 \mathrm{~S}$ rRNA gene. Microbiological Research 207:91-99 DOI 10.1016/j.micres.2017.11.010.

Arizza V, Vecchioni L, Caracappa S, Sciurba G, Berlinghieri F, Gentile A, Persichetti MF, Arculeo M, Alduina R. 2019. New insights into the gut microbiome in loggerhead sea turtles Caretta caretta stranded on the Mediterranean coast. PLoS One 14:e0220329 DOI 10.1371/journal.pone.0220329.

Buchon N, Broderick NA, Lemaitre B. 2013. Gut homeostasis in a microbial world: insights from Drosophila melanogaster. Nature Reviews Microbiology 11:615-626 DOI 
10.1038/nrmicro3074.

510 Cadi A, Delmas V, Prevot A-C, Joly P, Pieau C, Girondot M. 2004. Successful reproduction of the introduced slider turtle (Trachemys scripta elegans) in the South 
529 Colonization of probiotic bacteria and its impact on ornamental fish Puntius

530 conchonius. Journal of Environmental Biology 33:551-555 DOI

$531 \quad$ 10.1016/j.enpol.2012.01.056.

532

533

534

535

536

537

Du S, Zhang WF, Shi HT. 2013. 16S rRNA-base analysis of Bacteroidetes and Firmicutes diversity in the microbial flora of the red-eared slider (Trachemys scripta elegans) intestinal tract. Genomics and Applied Biology 32:700-706 (In Chinese).

Edgar RC. 2010. Search and clustering orders of magnitude faster than BLAST. Bioinformatics 26:2460-2461 DOI 10.1093/bioinformatics/btq461.

Egerton S, Culloty S, Whooley J, Stanton C, Ross R. 2018. The gut microbiota of marine fish. Frontiers in Microbiology 9:873 DOI 10.3389/fmicb.2018.00873.

\section{Fernando SC, Purvis HT, Najar FZ, Sukharnikov LO, Krehbiel CR, Nagaraja TG, Roe} BA, DeSilva U. 2010. Rumen microbial population dynamics during adaptation to a high-grain diet. Applied and Environmental Microbiology 76:7482-7490 DOI 10.1128/aem.00388-10.

Fugate HM, Kapfer JM, and McLaughlin RW. 2020. Analysis of the microbiota in the fecal material of painted turtles (Chrysemys picta). Current Microbiology 77:11-14 DOI 10.1007/s00284-019-01787-5.

Fugate HM, Kapfer JM, and McLaughlin RW. 2020. Analysis of the microbiota in the fecal material of painted turtles (Chrysemys picta). Current Microbiology 77:11-14 DOI 10.1007/s00284-019-01787-5. 
549 Gao S, Pan LQ, Zhang MZ, Huang F, Zhang MY, He ZY. 2020. Screening of bacterial

550 strains from the gut of Pacific White Shrimp (Litopenaeus vannamei) and their

551 efficiencies in improving the fermentation of soybean meal. FEMS Microbiology

Hong YH, Huang Y, Wu S, Yang X, Dong Y, Xu D, Huang Z. 2020. Effects of imidacloprid on the oxidative stress, detoxification and gut microbiota of Chinese mitten crab, Eriocheir sinensis. Science of the Total Environment 729:138276 DOI 10.1016/j.scitotenv.2020.138276.

Kanehisa M. 2019. Toward understanding the origin and evolution of cellular organisms. Protein Science 28:1947-1951 DOI 10.1002/pro.3715.

Kartzinel TR, Hsing JC, Musili PM, Brown BRP, Pringle RM. 2019. Covariation of diet and gut microbiome in African megafauna. Proceedings of the National Academy of Sciences of the United States of America 116:23588-23593 DOI 
10.1073/pnas.1905666116.

570

571

572

Keenan SW, Engel AS, Elsey RM. 2013. The alligator gut microbiome and implications for archosaur symbioses. Scientific Reports 3:2877 DOI 10.1038/srep02877.

Kohl KD, Brun A, Magallanes M, Brinkerhoff J, Laspiur A, Acosta JC, CaviedesVidal E, Bordenstein SR. 2017. Gut microbial ecology of lizards: insights into diversity in the wild, effects of captivity, variation across gut regions and transmission. Molecular Ecology 26:1175-1189 DOI 10.1111/mec.13921.

\section{Langille MGI, Zaneveld J, Caporaso JG, McDonald D, Knights D, Reyes JA,} Clemente JC, Burkepile DE, Vega Thurber RL, Knight R, Beiko RG, Huttenhower C. 2013. Predictive functional profiling of microbial communities using 16S rRNA marker gene sequences. Nature Biotechnology 31:814-821 DOI 10.1038/nbt.2676.

Lankau EW, Hong PY, Mackie RI. 2012. Ecological drift and local exposures drive enteric bacterial community differences within species of Galápagos iguanas. Molecular Ecology 21:1779-1788 DOI 10.1111/j.1365-294X.2012.05502.x.

Ley RE, Bäckhed F, Turnbaugh P, Lozupone CA, Knight RD, Gordon JI. 2005. Obesity alters gut microbial ecology. Proceedings of the National Academy of Sciences of the United States of America 102:11070-11075 DOI 10.1073/pnas.0504978102.

Ley RE, Hamady M, Lozupone C, Turnbaugh PJ, Ramey RR, Bircher JS, Schlegel ML, Tucker TA, Schrenzel MD, Knight R, Gordon JI. 2008. Evolution of mammals 
and their gut microbes. Science 320:1647-1651 DOI 10.1126/science.1155725.

590 Li H, Zhou ZS, Wu T, Wu YQ, Ji X. 2013. Do fluctuations in incubation temperature

affect hatchling quality in the Chinese soft-shelled turtle Pelodiscus sinensis?

Aquaculture 406/407:91-96 DOI 10.1016/j.aquaculture.2013.05.011.

Lin M, Zeng CX, Li ZQ, Ma Y, Jia XQ. 2019. Comparative analysis of the composition and function of fecal - gut bacteria in captive juvenile Crocodylus siamensis between healthy and anorexic individuals. MicrobiologyOpen:1-10 DOI 10.1002/mbo3.929.

Ma K, Shi H-T. 2017. Red-eared slider Trachemys scripta elegans (Wied-Neuwied). In: Wan F-H, Jiang M-X, and Zhan A-B, eds. Biological invasions and its management in China. Netherlands: Springer Press, 49-76 DOI 10.1007/978-981-10-3427-5_4.

McDermid KJ, Kittle RP, Veillet A, Plouviez S, Muehlstein L, Balazs GH. 2020. Identification of gastrointestinal microbiota in Hawaiian green turtles (Chelonia mydas). Evolutionary Bioinformatics 16:1176934320914603 DOI 10.1177/1176934320914603.

Mclaughlin RW, Cochran PA, and Dowd SE. 2015. Metagenomic analysis of the gut microbiota of the timber rattlesnake, Crotalus horridus. Molecular Biology Reports 42:1187-1195 DOI 10.1007/s11033-015-3854-1.

Md Zoqratt MZH, Eng WWH, Thai BT, Austin CM, Gan HM. 2018. Microbiome analysis of Pacific white shrimp gut and rearing water from Malaysia and Vietnam: implications for aquaculture research and management. PeerJ 6:e5826 DOI 
10.7717/peerj.5826.

610

611

612

613

614

615

616

617

618

619

620

621

622

623

624

625

626

627

628

Mendoza MLZ, Roggenbuck M, Manzano Vargas K, Hansen LH, Brunak S, Gilbert

MTP, Sicheritz-Pontén T. 2018. Protective role of the vulture facial skin and gut

microbiomes aid adaptation to scavenging. Acta Veterinaria Scandinavica 60:61 DOI

10.1186/s13028-018-0415-3.

Meziti A, Mente E, Kormas KA. 2012. Gut bacteria associated with different diets in reared Nephrops norvegicus. Systematic and Applied Microbiology 35:473-482 DOI 10.1016/j.syapm.2012.07.004.

Mueller S, Saunier K, Hanisch C, Norin E, Alm L, Midtvedt T, Cresci A, Silvi S, Orpianesi C, Verdenelli MC, Clavel T, Koebnick C, Zunft HJF, Dore J, Blaut M. 2006. Differences in fecal microbiota in different European study populations in relation to age, gender, and country: a cross-sectional study. Applied and Environmental Microbiology 72:1027-1033 DOI 10.1128/aem.72.2.1027-1033.2006.

Nelson TM, Rogers TL, Carlini AR, Brown MV. 2013. Diet and phylogeny shape the gut microbiota of Antarctic seals: a comparison of wild and captive animals. Environmental Microbiology 15:1132-1145 DOI 10.1111/1462-2920.12022.

Nuriel-Ohayon M, Neuman H, Koren O. 2016. Microbial changes during pregnancy, birth, and infancy. Frontiers in Microbiology 7:1031 DOI 10.3389/fmicb.2016.01031.

Oksanen J, Blanchet FG, Kindt R, Legendre P, Minchin PR, O'Hara RB, Simpson GL, Solymos P, Stevens MHH, Wagner H. 2013. Community ecology package. 
http://cran.r-project.org.

630

631

632

633

Peng Q, Chen Y, Ding L, Zhao Z, Yan P, Storey KB, Shi HT, Hong ML. 2020. Early-life intestinal microbiome in Trachemys scripta elegans analyzed using 16S rRNA sequencing. PeerJ 8:e8501 DOI 10.7717/peerj.8501.

Qin JJ, Li RQ, Raes J, Arumugam M, Burgdorf KS, Manichanh C, Nielsen T, Pons N, Levenez F, Yamada T, Mende DR, Li JH, Xu JM, Li SC, Li DF, Cao JJ, Wang B, Liang HQ, Zheng HS, Xie YL, Tap J, Lepage P, Bertalan M, Batto JM, Hansen T, Le Paslier D, Linneberg A, Nielsen HB, Pelletier E, Renault P, Sicheritz-Ponten T, Turner K, Zhu HM, Yu C, Li ST, Jian M, Zhou Y, Li YR, Zhang XQ, Li SG, Qin N, Yang HM, Wang J, Brunak S, Dore J, Guarner F, Kristiansen K, Pedersen O, Parkhill J, Weissenbach J, Bork P, Ehrlich SD, Wang J. 2010. A human gut microbial gene catalogue established by metagenomic sequencing. Nature 464:5965 DOI 10.1038/nature08821.

Quast C, Pruesse E, Yilmaz P, Gerken J, Schweer T, Yarza P, Peplies J, Glöckner FO. 2012. The SILVA ribosomal RNA gene database project: improved data processing and web-based tools. Nucleic Acids Research 41:D590-D596 DOI $10.1093 /$ nar/gks1219.

R Development Core Team. 2020. R: a language and environment for statistical computing. $\mathrm{R}$ foundation for statistical computing, Vienna, Austria, http://www.Rproject.org. 
649 Rowland I, Gibson G, Heinken A, Scott K, Swann J, Thiele I, Tuohy K. 2018. Gut

650 microbiota functions: metabolism of nutrients and other food components.

651 European Journal of Nutrition 57:1-24 DOI 10.1007/s00394-017-1445-8.

652

654

655

656

657

658

659

660

661

662

663

664

665

666

667

668

Rungrassamee W, Klanchui A, Maibunkaew S, Chaiyapechara S, Jiravanichpaisal P, Karoonuthaisiri N. 2014. Characterization of intestinal bacteria in wild and domesticated adult black tiger shrimp (Penaeus monodon). PLoS ONE 9:e91853 DOI 10.1371/journal.pone.0091853.

Scheelings TF, Moore RJ, Van TTH, Klaassen M, Reina RD. 2020. Microbial symbiosis and coevolution of an entire clade of ancient vertebrates: the gut microbiota of sea turtles and its relationship to their phylogenetic history. Animal Microbiome 2:17 DOI 10.1186/s42523-020-00034-8.

Schloss PD, Westcott SL, Ryabin T, Hall JR, Hartmann M, Hollister EB, Lesniewski RA, Oakley BB, Parks DH, Robinson CJ, Sahl JW, Stres B, Thallinger GG, Van Horn DJ, Weber CF. 2009. Introducing mothur: open-source, platformindependent, community-supported software for describing and comparing microbial communities. Applied and Environmental Microbiology 75:7537-7541 DOI 10.1128/AEM.01541-09.

Scott KA, Ida M, Peterson VL, Prenderville JA, Moloney GM, Izumo T, Murphy K, Murphy A, Ross RP, Stanton C, Dinan TG, Cryan JF. 2017. Revisiting Metchnikoff: Age-related alterations in microbiota-gut-brain axis in the mouse. 
Brain Behav Immun 65:20-32 DOI 10.1016/j.bbi.2017.02.004.

670

671

672

673

674

675

676

677

678

679

680

681

682

683

684

685

686

687

688

Segata N, Izard J, Waldron L, Gevers D, Miropolsky L, Garrett WS, Huttenhower C.

2011. Metagenomic biomarker discovery and explanation. Genome Biology 12:R60

DOI 10.1186/gb-2011-12-6-r60.

Songjinda P, Nakayama J, Tateyama A, Tanaka S, Tsubouchi M, Kiyohara C,

Shirakawa T, Sonomoto K. 2007. Differences in developing intestinal microbiota

between allergic and non-allergic infants : a pilot study in Japan. Bioscience,

Biotechnology, and Biochemistry 71:2338-2342 DOI 10.1271/bbb.70154.

Strandwitz P, Kim KH, Terekhova D, Liu JK, Sharma A, Levering J, McDonald D,

Dietrich D, Ramadhar TR, Lekbua A, Mroue N, Liston C, Stewart EJ, Dubin MJ,

Zengler K, Knight R, Gilbert JA, Clardy J, Lewis K. 2019. GABA-modulating

bacteria of the human gut microbiota. Nature Microbiology 4:396-403 DOI

10.1038/s41564-018-0307-3.

Suzuki TA, Worobey M. 2014. Geographical variation of human gut microbial composition. Biology Letters 10:20131037 DOI 10.1098/rsbl.2013.1037.

Tang GS, Liang XX, Yang MY, Wang TT, Chen JP, Du WG, Li H, Sun BJ. 2020.

Captivity influences gut microbiota in crocodile lizards (Shinisaurus crocodilurus).

Frontiers in Microbiology 11:1-12 DOI 10.3389/fmicb.2020.00550.

Turnbaugh PJ, Ley RE, Mahowald MA, Magrini V, Mardis ER, Gordon JI. 2006. An

obesity-associated gut microbiome with increased capacity for energy harvest. 
Nature 444:1027-1031 DOI 10.1038/nature05414.

690

691

692

693

694

695

696

697

698

Vatanen T, Plichta DR, Somani J, Münch PC, Arthur TD, Hall AB, Rudolf S, Oakeley EJ, Ke X, Young RA, Haiser HJ, Kolde R, Yassour M, Luopajärvi K, Siljander H, Virtanen SM, Ilonen J, Uibo R, Tillmann V, Mokurov S, Dorshakova N, Porter JA, McHardy AC, Lähdesmäki H, Vlamakis H, Huttenhower C, Knip M, Xavier RJ. 2019. Genomic variation and strain-specific functional adaptation in the human gut microbiome during early life. Nature Microbiology 4:470-479 DOI 10.1038/s41564-018-0321-5.

Videvall E, Song SJ, Bensch HM, Strandh M, Engelbrecht A, Serfontein N, Hellgren O, Olivier A, Cloete S, Knight R, Cornwallis CK. 2019. Major shifts in gut microbiota during development and its relationship to growth in ostriches. Molecular Ecology 28:2653-2667 DOI 10.1111/mec.15087.

Wang CH, Zhou YF, Lv DW, Ge Y, Li H, You Y. 2019. Change in the intestinal bacterial community structure associated with environmental microorganisms during the growth of Eriocheir sinensis. MicrobiologyOpen 8:e00727 DOI 10.1002/mbo3.727.

Wang W, Liu YB, Yang YS, Wang AZ, Sharshov K, Li Y, Cao MY, Mao PZ, Li LX. 2018. Comparative analyses of the gut microbiota among three different wild geese species in the genus Anser. Journal of Basic Microbiology 58:543-553 DOI 10.1002/jobm.201800060.

Wei HL, Li X, Tang L, Yao HZ, Ren ZM, Wang CL, Mu CK, Shi C, Wang H. 2020. 16 S 
709

710

711

712

rRNA gene sequencing reveals the relationship between gut microbiota and ovarian development in the swimming crab Portunus trituberculatus. Chemosphere 254:126891 DOI 10.1016/j.chemosphere.2020.126891.

Wei HL, Wang H, Tang L, Mu CK, Ye CY, Chen LZ, Wang CL. 2019. High-throughput sequencing reveals the core gut microbiota of the mud crab (Scylla paramamosain) in different coastal regions of southern China. BMC Genomics 20:829 DOI 10.1186/s12864-019-6219-7.

Xing MX, Hou ZH, Yuan JB, Liu Y, Qu YM, and Liu B. 2013. Taxonomic and functional metagenomic profiling of gastrointestinal tract microbiome of the farmed adult turbot (Scophthalmus maximus). FEMS Microbiology Ecology 86:432-443 DOI 10.1111/1574-6941.12174.

Ye L, Amberg J, Chapman D, Gaikowski M, Liu WT. 2014. Fish gut microbiota analysis differentiates physiology and behavior of invasive Asian carp and indigenous American fish. ISME Journal 8:541-551 DOI 10.1038/ismej.2013.181.

Yuan ML, Dean SH, Longo AV, Rothermel BB, Tuberville TD, Zamudio KR. 2015. Kinship, inbreeding and fine-scale spatial structure influence gut microbiota in a hindgut-fermenting tortoise. Molecular Ecology 24:2521-2536 DOI 10.1111/mec.13169.

Zhang H, Liao X, Sparks JB, Luo XM. 2014. Dynamics of gut microbiota in autoimmune lupus. Applied and Environmental Microbiology 80:7551-7560 DOI 

(Andrias davidianus). MicrobiologyOpen 8:e00778 DOI 10.1002/mbo3.778. populations of Chinese rhesus macaques (Macaca mulatta), implying an adaptation to high-altitude environment. Microbial Ecology 76:565-577 DOI 10.1007/s00248-0181146-8. 10.1002/mbo3.1095. affects diversity, abundance and functional pathways of gut microbiota in the northern grass lizard Takydromus septentrionalis. MicrobiologyOpen 9:e1095 DOI 
744 Figure legends

745 Figure 1 Relative abundances of the gut microbiota at the phylum (A), class (B), order

746 (C), and family (D) levels in Chinemys reevesii (CR) and Trachemys scripta elegans

747 (TSE). One color indicates one taxon in each plot. The color for others in Plot D

748 indicates all other families not listed in the plot.

749 Figure 2 The differences in relative abundance of gut microbiota between Chinemys

750 reevesii and Trachemys scripta as shown by linear discriminatory analysis (LDA)

751 scores. Letters p, c, o and f indicate phylum, class, order and family, respectively. See

752 Figure 1 for species abbreviations.

753 Figure 3 Fecal microbial diversity in the two turtle species. Results of principal

754 coordinates analysis (PCoA) and non-metric multidimensional scaling (MDS) of Bray-

755 Curtis distance matrix for bacterial abundance are shown in plots A and B, respectively.

756 See Figure 1 for species abbreviations.

757 Figure 4 Gene functional categories based on 16S RNA in the gut microbiota at top

758 (A), second (B) and third (C) levels of relative abundance. One color indicates one

759 gene function. Detailed descriptions are shown on the right side of each plot. The colors

760 for others in Plots B and C indicate all other gene functions not listed in these two plots.

761 See Figure 1 for species abbreviations. 


\section{Figure 1}

Figure 1 Relative abundances of the gut microbiota at the phylum (A), class (B), order (C), and family (D) levels in Chinemys reevesii (CR) and Trachemys scripta elegans (TSE).

Relative abundances of the gut microbiota at the phylum (A), class (B), order (C), and family (D) levels in Chinemys reevesii (CR) and Trachemys scripta elegans (TSE). One color indicates one taxon in each plot. The color for others in Plot D indicates all other families not listed in the plot.

A

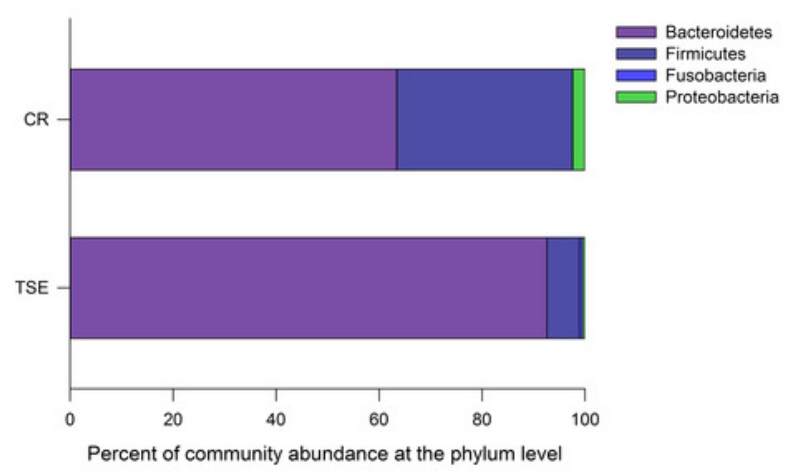

C

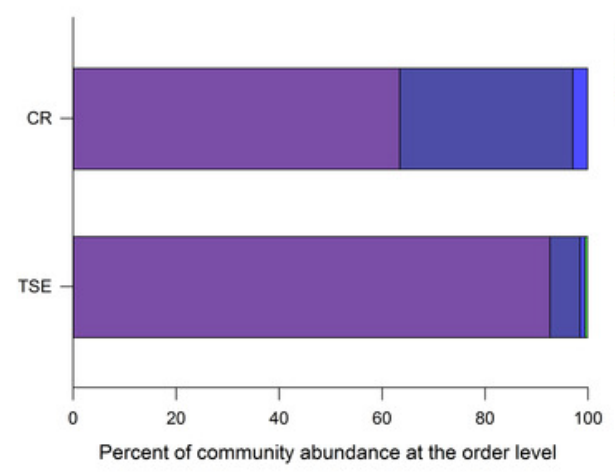

B

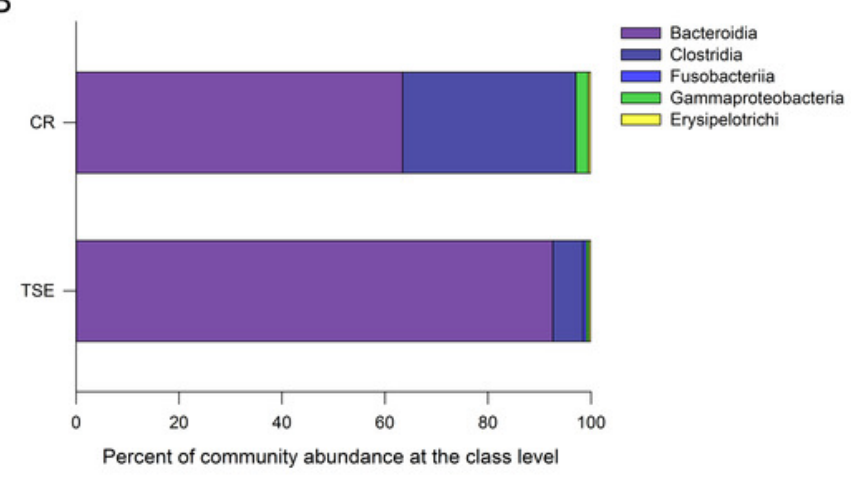

D

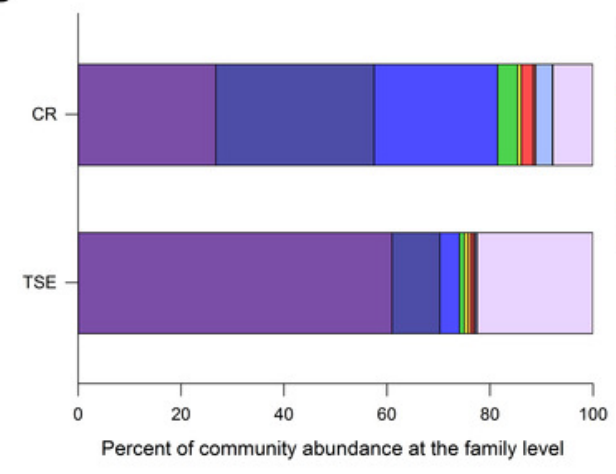

Porphyromonadaceae Bacteroidaceae Lachnospiracea Clostridiaceae Ruminococcaceae Fusobacteriaceae Enterobacteriaceae S24-8 Aeromonadaceae Others 
Figure 2

Figure 2 The differences in relative abundance of gut microbiota between Chinemys reevesii and Trachemys scripta as shown by linear discriminatory analysis (LDA) scores.

The differences in relative abundance of gut microbiota between Chinemys reevesii and Trachemys scripta as shown by linear discriminatory analysis (LDA) scores. Letters $p, c, 0$ and f indicate phylum, class, order and family, respectively. See Figure 1 for species abbreviations. 


\section{Cladogram}

CR
TSM

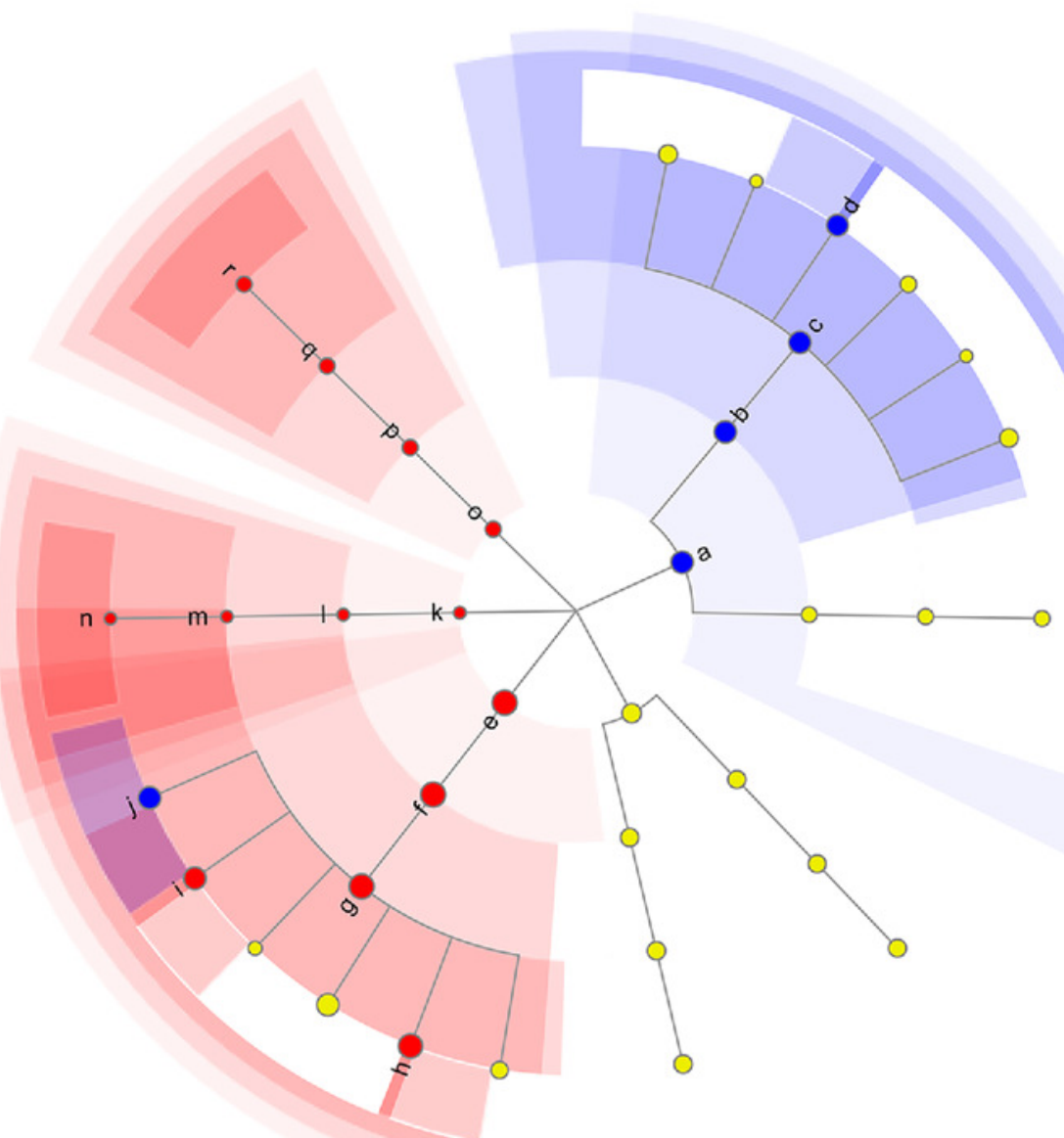

- a : p_Firmicutes

- b:c_Clostridia

- c: o-Clostridiales

- d:f_Lachnospiraceae

- e : p_Bacteroidetes

- $\mathrm{f}$ : c_Bacteroidia

- $g: \bar{c}$ Bacteroidales

- h:f_Porphyromonadaceae

- i : f_unclassified_o_Bacteroidales
- j : f_Bacteroidaceae

- $\mathrm{k}:$ p_unclassified_k_norank_d_Bacteria

- I : c_unclassified_k_norank_d_Bacteria

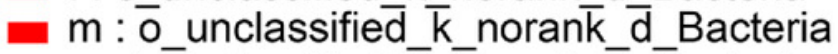

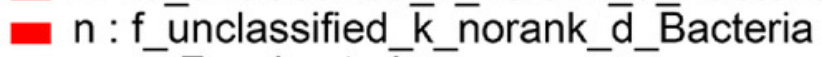

- o:p_Fusobacteria

- p : c_Fusobacteriia

- q:o_Fusobacteriales

- r:f_Fusobacteriaceae 
Figure 3

Figure 3 Fecal microbial diversity in the two turtle species.

Fecal microbial diversity in the two turtle species. Results of principal coordinates analysis (PCoA) and non-metric multidimensional scaling (MDS) of Bray-Curtis distance matrix for bacterial abundance are shown in plots A and B, respectively. See Figure 1 for species abbreviations.

A

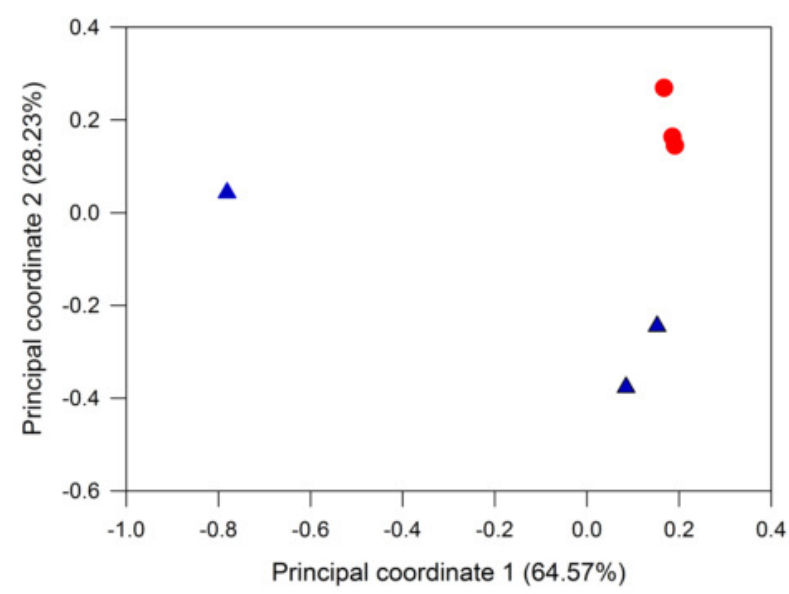

B NMDS on OTU level

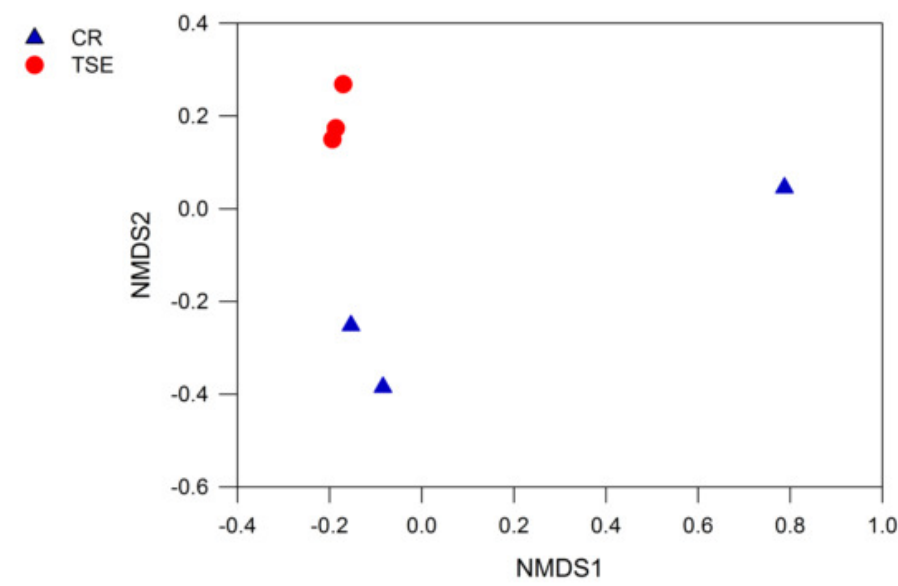


Figure 4

Figure 4 Gene functional categories based on 16S RNA in the gut microbiota at top (A), second $(B)$ and third $(C)$ levels of relative abundance.

Gene functional categories based on 16S RNA in the gut microbiota at top (A), second (B) and third (C) levels of relative abundance. One color indicates one gene function. Detailed descriptions are shown on the right side of each plot. The colors for others in Plots B and C indicate all other gene functions not listed in these two plots. See Figure 1 for species abbreviations. 
A

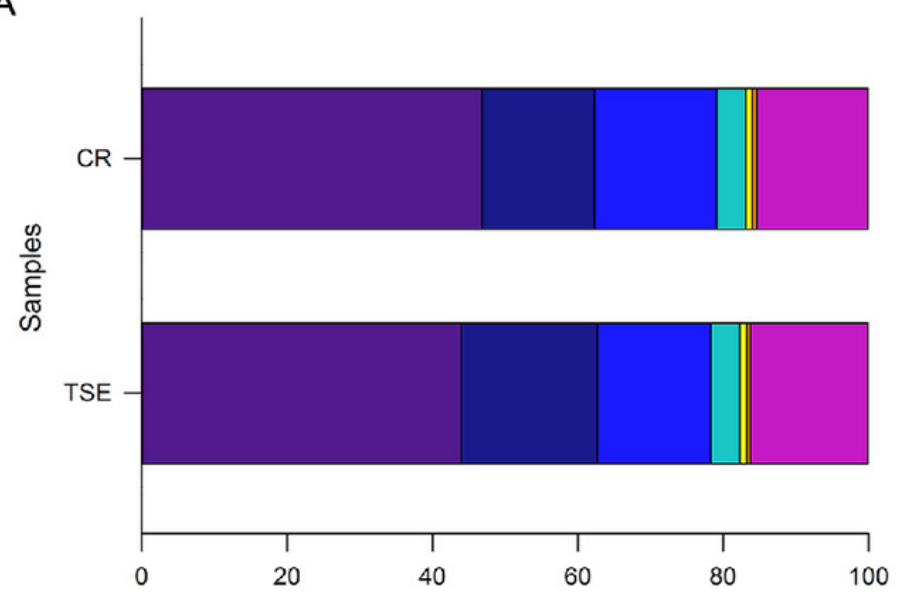

Relative abundance of KEGG gene pathways

B

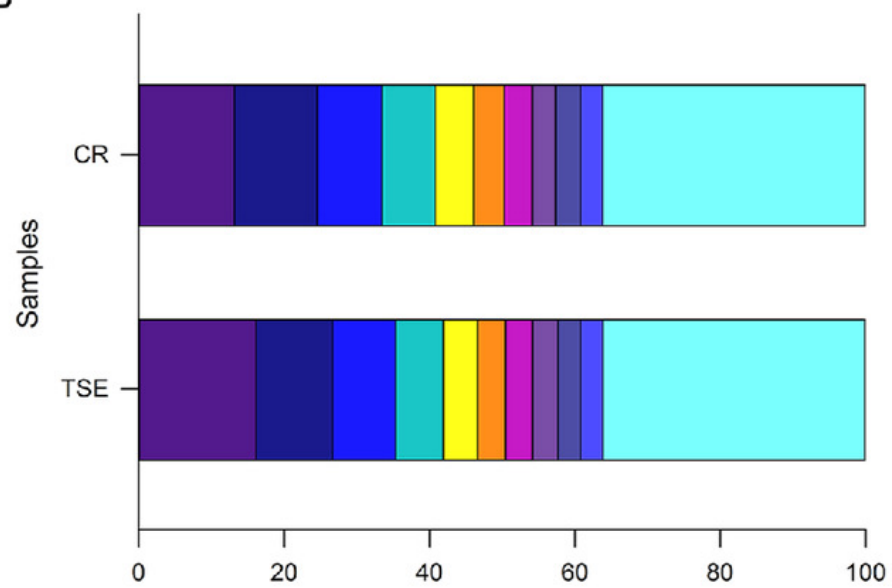

Relative abundance of KEGG gene pathways

C

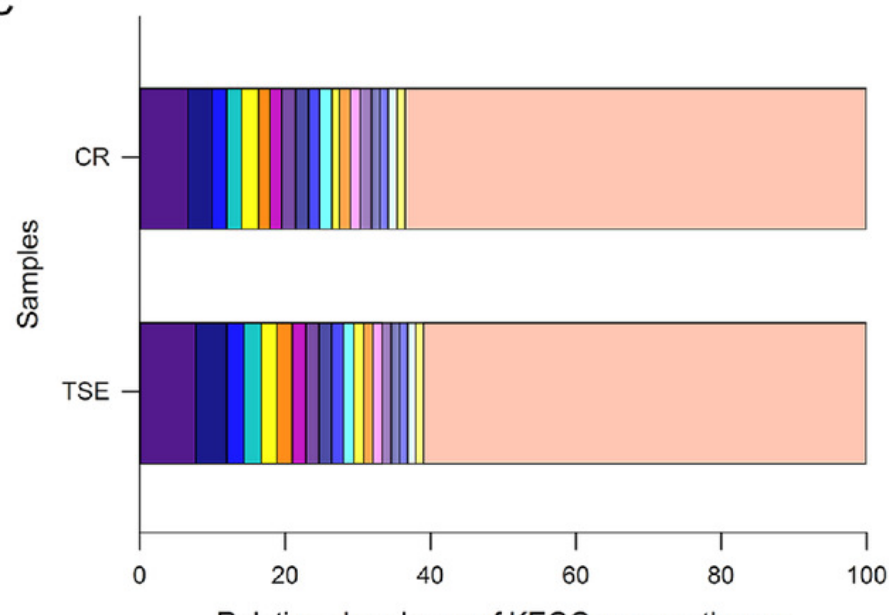

Relative abundance of KEGG gene pathways
Metabolism

Environmental Information Processing

Genetic Information Processing

$\square$ Cellular Processes

$\square$ Human Diseases

$\square$ Organismal Systems

Unclassified

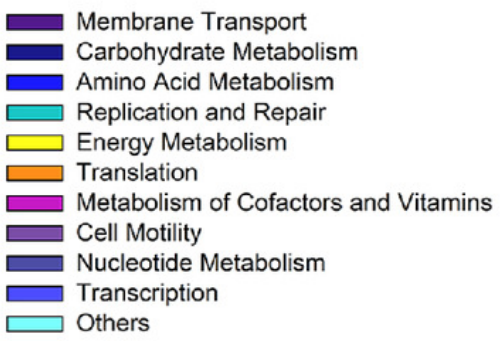

